\title{
Digital Education and Personal Safety
}

\author{
Liudmila Baeva ${ }^{1, *}$ \\ ${ }^{1}$ Astrakhan State University, Astrakhan, Russia \\ *Corresponding author. Email: baevaludmila@mail.ru
}

\begin{abstract}
The article concerns the process of digitalization of education in the context of the analysis of the personal safety problem. The aim of the study was to assess the impact of digitalization of education on a person in terms of its safety and to diagnose emerging challenges in education related to accelerated digital transition in 2020 . The empirical sources for the study were as following: an expert survey of teachers, focus group interviews, and an online survey with teachers of general and vocational education schools. The results of the conducted empirical and theoretical research allowed the author to identify the key problems that arise on the way to digitalization of education, to systematize them and to put forward recommendations to reverse negative scenarios. The obtained results can be used by researchers of digital society issues, teachers, administrators of information networks, and information security specialists in education.
\end{abstract}

Keywords: Digitalization on education, E-culture, Safety, Risks, Safe environment.

\section{INTRODUCTION}

In the context of digital transformations of society and its institutions, social safety is one of the priorities for sustainability of systems. In this regard, safety is associated with protection of social and cultural systems from possible deformations, which could lead to violation of rights and individual freedom as a result of information and psychological impact of modern electronic resources. Ensuring safety and sustainability in a modern open society is becoming one of the leading tasks relating to the education system. Digitalization of education is spreading from universities and massive open online courses to school and becoming a general trend. This process can radically change the essence of education as a space for communication, dialogue, and socialization forming not only knowledge but also social skills. The goal of the study is to systematize and characterize social and anthropological risks of digitalization of education and communication of the youth. The issues are based on the usage of axiological approach to studying safety principles for the communication and educational environment as a phenomenon of e-culture.

E-culture, or digital culture, is, first of all, a new sphere of human activity associated with the creation of electronic copies of spiritual and material objects, as well as the creative work of virtual objects of science, communication, art, and education. Nowadays, e-culture is an interdisciplinary concept having connotations in philosophy, cultural studies, sociology, education, political science, economics, and of course information technologies. Its subjects and creators are scientists, programmers, artists, representatives of mass media and average users of information systems creating electronic forms of selfrepresentation and self-manifestation in the global Internet by technological means. The most important characteristics of e-culture are transparency, globality, and accessibility for every user. Moreover, every subject can become both a user and a creator, being rather free in creative work and not having strict limitations. The distinctive features of e-culture are a digital form, virtuality, accessibility, openness for the members of information community (those who have electronic resources), remotability, physical distance from a subject, activity in gaining access to electronic information, opportunity to participate in developing the information content from any point of the information space, liberality, linguistic description, absence of strict rules and norms, dominance of visual things over conceptual ones, innovativeness, technocracy, and a high speed of changing [2]. 
Informatization of education adapts a person to a new type of culture which has its own ethics, rules, and regulations. Thus, it develops a person able to realize their need for information, search for the required information and find it promptly, process that information and assess, select, classify, or categorize it; in the end, that person is able to create new information that meets the criteria of knowledge.

On the one hand, education in the context of virtualization and distance technologies gives individuals more freedom to choose, thus allowing them to master different computer programs, use previously unavailable data, study at home or in an office, etc. However, education as a traditional system that has not changed for thousands of years, existed as a way to transfer knowledge from one person to another. The modern education has rejected this model and shifted to pluralistic and extra-personal education "from a person to information resources" (Ackoff, R. L., 1989). This also changes cognitive processes: the value of education is progressively decreasing but the value of information is increasing.

Social risks and problems in this sphere may be due to: reduction of faculty staff; dysfunction of traditional schools and universities; virtualization and lack of feedback from students; weakened role of the upbringing and value-forming functions of education; monopolized educational services; a real opportunity to mesmerize students by means of manipulative techniques; loss of opportunities to socialize students; reduction of quality of education owing to the absence of the individual approach to students, etc. At the same time, a person who is forced to live in the electronic culture and media sector needs to get the skill of working online. Open education enables a person thereby to adapt to the information world, gives them the opportunity to learn communication, information ethics, security, and culture. Owing to the current progress of these tendencies, the development of open-education systems and techniques aimed at people with different abilities and different needs is becoming essential. Social and humanitarian knowledge is facing a complicated task to preserve interpersonal communication between a student and a teacher, teamwork and leadership skills in young people to provide their socialization, form cultural and ethical values of new generations, and overcome destructive, self-destructive, and aggressive tendencies that are typical of a human way of life beyond a human society. Therefore, it is necessary to develop principles and imperatives of education in accordance with the new paradigm.

\section{ISSUES OF THE IMPACT OF EDUCATION DIGITALIZATION}

The problem of assessing the impact of digitalization on the education system in terms of students' personal security is relatively new and understudied. Since the 2000s, the topic of digitalization of education has been closely related to the assessment of both positive and negative effects of this process. One of the first large-scale works in this area was the monograph by $\mathrm{D}$. Johnston and L. Baker Assessing the Impact of Technology in Teaching and Learning, published in Michigan in 2002 [6]. This study presented the results of the application of online teaching technologies that were innovative for that time and assessed their impact in various fields: cognitive and affective spheres, educational outcomes of teaching adults, changes in pedagogy, improving teachers' technological skills, and technological integration in studying.

The positive impact of digital education on the development of students' skills is presented in the studies by M. Ragard [9], G. Gable, D. Sedera, and T. Chan [4], R. M Tawafak, A. B. Romli, R. Arshah, and R. A. Almaroof [10], etc. These authors stress that digital learning allows students to widely use the opportunities of individualization, interactivity, visualization, gamification of studying, and creates great opportunities for education management based on artificial intelligence, and big data concerning learning outcomes and problems.

Meanwhile, some studies identify a number of significant problems and risks in the application of digital learning associated with a decrease in motivation to learn, decreasing cognitive functions, and harmful effects on interpersonal communication skills, healthy lifestyle, etc. For example, the problems of a healthy lifestyle distortion were studied in the work of $\mathrm{R}$. Mustafaoğlu [8]. The study by Y. Alghamdi [1] showed that the introduction of digital educational technologies at young age could lead children to social exclusion, and cause depression, serious mental and physical illnesses and disorders.

The study of negative effects of mobile learning conducted by analysing the cognitive load and students' learning ourcomes is presented in the article by Hui-Chun Chu [3]. The author 
substantiates the thesis that without proper support, educational progress of students using the existing online learning strategies (that are considered to be effective) can be disappointing. Besides, negative consequences can be caused by a large cognitive load due to the incorrect design of training.

Thus, modern research shows that assessing the impact of digital education on a person is crucial to achieve a positive effect. Assessment of possible risks in the field of digitalization of secondary and high school education should be a starting point for building a secure communicative and educational environment.

\section{THREATS TO DIGITAL EDUCATIONAL UNDER CONDITIONS OF LOCKDOWN}

Year 2020 was a turning point for the world as a whole and for the education system in particular, which was forcedly transferred to a distance format in order to reduce the spread of the coronavirus infection. This process was uneven and showed that a significant part of schools and universities were not ready for such a transformation. However, in the context of self-organization, the education system rapidly began to adapt to new conditions, and by the second semester considerable experience had already been accumulated in methodical, organizational and educational activities, as well as, in some cases, in psychological and social support for students.

As the education process became conditioned by the peculiarities of virtual communication and shifted to the digital environment, certain social threats also emerged. The practice of general transition to online learning showed the critical threats that were associated with violation of personal security and human rights in the digital environment, with the possibility of cyberattacks, transmission of classified information, and the spread of fakes and cyber fraud, which in these conditions receive new impulses and forms of manifestation.

The research focused on assessing the risks of digitalization of education. The author of the article studied them based on situational analysis of the year 2020 and empirical surveys of teachers of schools and vocational schools who faced new challenges for the first time. As an empirical method of research, the author chose an expert survey of educators who used digital technology in their job. Choosing the research method, the author faced the task of formulating hypotheses about the degree and directions of influence of digitalization on the learning process, as well as its potential risks for students. To solve this problem, "soft", qualitative research methods are best suited. They do not limit respondents to the strict framework of standardized tools, but provide freedom in expressing one's own opinion. Such was the reason for choosing this research method.

In 2020, within the framework of the project "Assessing the impact of digitalization of education on a person" conducted at the Astrakhan State University, two surveys and a series of focus groups with school and vocational school teachers of Astrakhan were organized and carried out to assess the problems encountered during the transition to the distance learning format (the sample consisted of 400 respondents, including teachers of schools and vocational schools of Astrakhan and Astrakhan region). The results of the first phase of the survey, showed that for teachers the most pressing risks were violations of authenticity and confidentiality. After the introduction of the self-isolation regime and the transition of schools to digital education, the situation changed dramatically: the teachers named digital inequality (low availability of technical resources) and violation of the integrity of educational content (more than $70 \%$ of respondents) as most serious risks. Risks to educational content were linked to a variety of factors, from fakes to cyberattacks. The results of the conducted surveys showed that the greatest fears among the pedagogical community in the implementation of the digital educational environment in the information sphere were caused by the risks of violation of confidentiality, content integrity and authenticity (over half of the respondents). The teachers also mentioned the risks in the legal sphere: the increase in cases of plagiarism among students, infringing of teachers' copyright on methodological or didactic materials, and a decrease in the level of unique educational material.

A series of focus groups with school teachers aimed at examining the main difficulties and risks encountered in the course of digitalization was held in June 2020. Three focus group interviews were organized according to the professional affiliation of the respondents and the use of digital educational technologies in their activities. During the processing of the results, the method of mind mapping was used; consequently, a mind map of teachers' opinions regarding the process of digitalization of education was drawn. 
The teachers identified the following risks faced in the digital learning environment and in the organization of educational activities:

- problems of accessible Internet connection and personal gadgets;

- introduction of undesirable, adult content into educational resources, general problems of information security violation;

- $\quad$ significant problems with organization of learning in the digital environment for primary and secondary schoolchildren who are not able to organize their workspace on their own;

- general decrease in learning achievements, motivation to study, concentration of attention during online classes, memorizing the material (possible reasons are associated with the loss of motor skills in writing, as well as an increased information flow), significant difficulties in absorbing educational material in several subjects;

- lack of teaching materials for learning in the digital environment;

- the risks of losing the opportunity to develop creative skills of students, as they can borrow ready-made samples in the digital environment;

- $\quad$ physical health and safety issues. Students have a dramatic increase in screen time, which affects eyesight, the musculoskeletal system, the nervous system, thus disrupting the healthy lifestyle of a student (and a teacher);

- possible risks to socialization and adaptation of students, weakening and even loss of the upbringing function of education associated with the transmission of values.

The results of surveys and focus groups were studied and formed the basis of the cluster theory of digitalization risks.

The following main risk-generating clusters were identified: information, cognitive, social, vital and addictive.

Information risks are associated with possible threats of negative information impact in the cyber environment. The main parameters of information threats to the educational process associated with the implementation of a unified digital educational environment, from the point of view of the main information security services, are: threats of violation of confidentiality, integrity, availability, authenticity and non-repudiation. The confidentiality parameter characterizes the ability to prevent the illegal distribution of personal information by participants in the educational process. The integrity parameter of educational content is associated with the impossibility of its illegitimate change, affecting the internal unity and logical connection in accordance with the requirements of the educational organization and federal standards. Accessibility to the digital learning environment means the possibility of unhindered entry to digital learning resources for all legitimate participants in the educational process. The authenticity parameter provides for the confirmation of identity and prevents impersonating other users. Non-repudiation is considered an important security parameter linked with preventing the possibility of refusing to create, receive or process information [7].

The cognitive cluster includes risks to cognitive processes. The following main types of possible cognitive risks of digitalization of the educational space were identified: 1) information overload in the cognitive sphere of students and keeping redundant information at the levels of attention, memory and thinking, causing fatigue and reducing motivation for learning; 2) serious transformation of students' consciousness, changing the content and structural/functional mechanisms of the dynamics of consciousness, expressed in the development of clip thinking; 3) devaluation of the possibilities of memory manifested in the domination of the short-term memory over the long-term one, and the alienation of individual memory from social (cultural) memory due to its depreciation and replacement by digital information resources; 4) decrease in the level of critical independent thinking revealing itself in the dominance of the emotional-figurative type of perception and thinking over the verbal-logical type and the devaluation of analytical abilities [11]

Vital risks are associated with a significant increase in screen time accompanying the digital transformation of education. The generalization of scientific sources and the results of focus groups surveys showed that the main risks were connected with emotional, mental and physical overload. The organs of vision, the musculoskeletal system, and the nervous system are the most vulnerable in this regard. Excessive screen time can be a source of physical and psychological hyperarousal for adolescents, sleep disorders, and emotional exhaustion, can provoke increased activation of the 
visual centres and lead to the development of spine diseases.

Social risks imply that the shift to the lifestyle of digital communication and educational environment for students can have certain riskgenerating effects forming difficulties with socialization, interpersonal communication, social skills, etc. Social risks have a delayed effect and can manifest themselves as communication further shifts to the digital environment. As noted by A.V. Grigoriev, one of the consequences of digitalization of education which reduces interpersonal communication may be the "desocialization of an individual expressed to different extent, namely, deterioration of communication skills, mismatch of rules and behavioural habits in society, weakening of the sense of community, belonging to a larger social group" $[5]^{\mathrm{p} .415}$. This becomes a manifestation of social exclusion, infringing of a person's involvement in social relations expressed through language, behavioural specifics, etc., and contributes to the increased alienation both between a teacher and students, and among students themselves. This alienation "leads to the development of other social risks of digitalization of education, namely, trolling and bullying". [5] $]^{\mathrm{p} .416}$

Addictive risks associated with various forms of electronic culture have been of particular concern in recent decades. An increase in students' screen time, and the transition of communication mainly to a virtual environment can contribute to the formation of new addictive disorders and forms of behaviour, such as addiction to social networks, gadgets and Internet communication.

The risks of digitalization of communication and education are manifested in different ways in certain conditions (social well-being, age characteristics, psychological aspects of personality, etc.), and therefore, a wide range of measures for social support of students should be introduced. Such support was of particular relevance in the crisis year of 2020, when all students found themselves in the conditions of a transition to digital learning.

\section{SAFE EDUCATIONAL DIGITAL ENVIRONMENT SYSTEM}

An essential step in digital transformation of the education system should be a system of a secure communication and educational environment, protected from threats to a person's rights and freedoms. It should properly protect their personal data, prevent addictive behaviour, loss of motivation for learning, decrease in cognitive skills, social adaptability and communication skills, etc. Most effective for an educational environment is the modular system of institutional and communication security based on the combination of modern theories of information security and digital culture. It includes the managerial, psychological, pedagogical, motivational, cognitive and ethical modules. Its composition requires not only tools to protect personal data of students, but also elements of recognition of students' psychological and emotional state. This allows monitoring risk-generating factors effectively, including those associated with programming behaviour (self-destructive, aggressive, addictive behaviour, loss of self-control, etc.). Another important element of a safe communication and educational environment is associated with selection of the most effective forms of learning, taking into account individual educational trajectories of students, in order to reduce the risks to the cognitive sphere. Such a system will help diagnose risks identified at the first stage of the project, and enable a teacher to construct psychological profiles of students and form risk groups and reference groups that can teach others (the principle of peer education). Thus, creation of a safe educational environment for digital education includes several main areas and modules:

- Improving technical sources of information and personal data protection;

- Creating safe communication for students;

- Ensuring a healthy lifestyle and protection from negative effects on the physical parameters of health;

- Protecting against addictions in a digital environment.

Each of the modules includes elements and forms of work related to ensuring safety of students in the face of increasing risks of a highly dynamic open informational environment, where each of participants is an active source of information and influences all participants in communication.

An important component in the system of digital education should also be a compulsory study of Ethics and Security in Digital Environment. The purpose of this academic discipline is to prevent risk-related consequences of information impact on students and to develop digital culture. The study of the basics of normative behaviour and communication in the digital environment can 
begin in high school. This course is complemented by the study of risk factors for negative information impact, the nature and manifestations of modern cyber threats and ways to counter them.

The tasks of the social support system should include, among other things:

- support for families that experience technology difficulties and creating equal educational opportunities;

- support of interpersonal communication and the formation of social skills of an individual;

- maintenance of a positive social and emotional state in virtual classrooms;

- prevention of deviant behaviour and social threats in the network interaction of students, including cyberbullying;

- development of codes of ethics for online education and training courses on the basics of safe behaviour in the cyber environment.

Social security and well-being depend on prevention of threats in the cyber environment that can intentionally harm an individual. Websites of educational organizations post materials on prevention and countermeasures against personal threats in the digital environment. However, an understanding of the relevance of these steps in a broad sense has not yet been achieved; meanwhile, a delay in the response to such challenges can lead to tragic consequences. Informing about existing threats alone is not sufficient to counter them; to counter socio-psychological cybersecurity threats, a system of measures in educational institutions is needed (along with the protection of information and personal data)

The system of social support for students as an important element of the digital learning environment should become one of the sources of prevention of possible deviations, addictions, destructive or self-destructive behaviour, as well as the basis for protecting an individual from threats in the cyber environment, violation of the rights, freedoms and dignity of an individual, and protection of personal data in order to maintain and develop the social environment of communication and to strengthen the skills of socialization of an individual both in the conditions of electronic culture and in real communication.

\section{CONCLUSION}

Thus, the following conclusions can be drawn regarding the assessment of security issues in a digital learning environment and their coverage in educational practices:

In the context of digital transformation, the learning system significantly changes the nature of social interaction of its subjects, breaking old ties and forming new ones. Social skills and interpersonal communication that are formed in the process of educational and extracurricular activities are extremely important for personality development and should also have a significant role in digital transformation. Another essential aspect of digital transformation is social well-being of a student, digital hygiene and the guarantee for the protection of their rights and freedoms in the digital environment. Interpersonal communication among students in virtual classrooms is being replaced by communication in social networks, which changes its nature and forms new social phenomena (including risk-generating ones associated with destructive information impact). In these conditions, social security in the digital educational environment is becoming one of the important components in this system which ensures the protection of the rights and freedoms of an individual.

The guarantee for social protection of individual rights in the information society should be associated, first of all, with the possibility of choosing a form of education (traditional, distance or hybrid), which should be taken into account when forming strategic documents in the field of education development at the national and regional levels. Students and parents should have the right to decide whether it is worth turning to distance learning in the digital environment, unless it is due to reasons of force majeure.

Another important parameter of social guarantees of human rights to education in the digital environment is the availability of resources, however, the responsibility to prove them which should not be completely shifted onto the families of students.

The digital learning environment created by order of modern governments in various countries is becoming a concentration of large volumes of educational materials, tests and assignments performed by students in many ways autonomously, the checking and marking of which will be supplemented with artificial intelligence in the near 
future. This will create conditions under which the social background that motivates learning and activity, and encourages moral experience and the dialogue with the Other (including the experience of communication in the intercultural sphere), is practically absent and replaced by the cyber environment, simulative community and communication. This requires the development of a system of social support and student support, designed to strengthen social and emotional skills, and moral forms of behaviour in the digital environment.

\section{AUTHORS' CONTRIBUTIONS}

This paper is independently completed by Liudmila Baeva.

\section{ACKNOWLEDGMENTS}

The author expresses gratitude to the Russian Foundation for Basic Research (RFBR) for supporting the project No. 19-29-14007 MK "Assessing the Impact of Digitalization of Educational and Social Spaces on a Person and Developing a Safe Communication and Educational Environment System", within the framework of which this article was prepared, and to reviewers for critical comments that helped to improve presentation of the research results.

\section{REFERENCES}

[1] Y. Alghamdi, Negative Effects of Technology on Children of Today. Oakland: Oakland University. 2016. doi: 10.13140/RG.2.2.35724.62089

[2] L. Baeva, E-Culture. In M. Khosrow-Pour (Ed.), Encyclopedia of Information Science and Technology: In 10 vol.: Vol. IX (3rd ed.). USA: IGI-Global.2014, pp. 6847-685

[3] H.-C. Chu, Potential Negative Effects of Mobile Learning on Students' Learning Achievement and Cognitive Load-A Format Assessment Perspective. Journal of Educational Technology \& Society, 17(1), 2014, pp. 332-344.

[4] G. Gable, D. Sedera and T. Chan, Reconceptualizing Information System Success: The IS-Impact Measurement Model. Journal of the Association for Information Systems, 9(7). 2008, doi: 10.17705/1jais.00164
[5] A. Grigorev, The social risks of digitalization of school education. Methodological and organizational issues of digital transformation of education in schools. Reports of the international conference. Krasnoyarsk. 2020, pp.

[6] J. Johnston, L. T. Barker, Assessing the impact of technology in teaching and learning: A sourcebook for evaluators. Ann Arbor, MI: Institute for Social Research, University of Michigan. 2002.

[7] V. Kuznetsova, I. Azhmukhamedov and L. Baeva, Analysis of Information Risks and Strategies for Protecting Schoolchildren from the Negative Consequences of Digitalization of Education. 244-249. Atlantis Press. 2020, doi: 10.2991/assehr.k.200509.045

[8] R. Mustafaoğlu, , E.Zirek, , Yasacı, Z., and A. Razak Özdinçler, The Negative Effects of Digital Technology Usage on Children's Development and Health. Addicta: The Turkish Journal on Addictions, 5(2). 2018, doi: 10.15805/addicta.2018.5.2.0051

[9] M. Ragad, Assessing the Impact of Technology Learning and Assessment Method on Academic Performance: Review Paper. EURASIA Journal of Mathematics, Science and Technology Education, 14(6), 2018 pp. 1305-1315.

[10] R. M Tawafak, A. B. Romli, R. Arshah and R. A. Almaroof, Assessing the Impact of Technology Learning and Assessment Method on Academic Performance: Review Paper. Eurasia Journal of Mathematics, Science and Technology Education, 14(6). 2018, doi: 10.29333/ejmste/87117

[11] S. A. Khrapov, (). Risks of Forming a "Technogenic (Digital) Identity" in the Digitalization of Educational Space. Vestnik of Tver State University. Series: Philosophy. (2), 2020, pp. 7-13. (In Russian). 